\title{
Effects of caffeine on the electrophysiological, cognitive and motor responses of the central nervous system
}

\author{
A.C. Deslandes ${ }^{1}$, \\ H. Veiga ${ }^{1}$, M. Cagy ${ }^{2}$, \\ R. Piedade ${ }^{1}$, F. Pompeu ${ }^{3}$ \\ and P. Ribeiro ${ }^{1,3,4}$
}

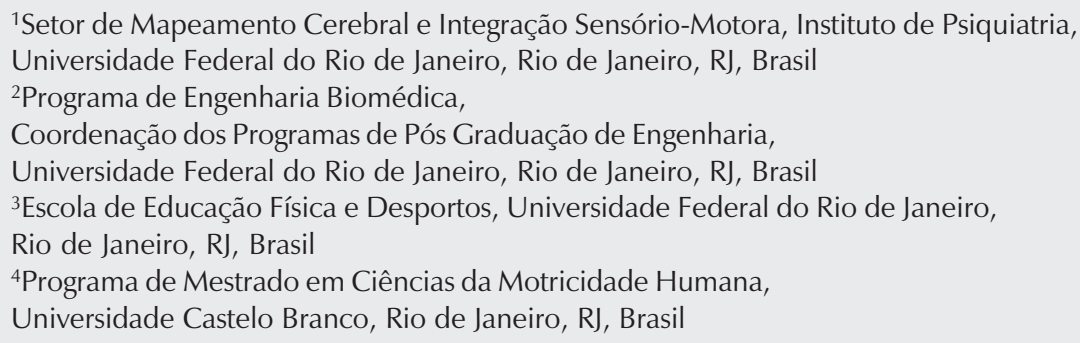

${ }^{1}$ Setor de Mapeamento Cerebral e Integração Sensório-Motora, Instituto de Psiquiatria, Universidade Federal do Rio de Janeiro, Rio de Janeiro, RJ, Brasil

${ }^{2}$ Programa de Engenharia Biomédica, Coordenação dos Programas de Pós Graduação de Engenharia, Universidade Federal do Rio de Janeiro, Rio de Janeiro, RJ, Brasil ${ }^{3}$ Escola de Educação Física e Desportos, Universidade Federal do Rio de Janeiro, Rio de Janeiro, RJ, Brasil

${ }^{4}$ Programa de Mestrado em Ciências da Motricidade Humana, Universidade Castelo Branco, Rio de Janeiro, RJ, Brasil

\section{Correspondence \\ A.C. Deslandes \\ Laboratório de Mapeamento \\ Cerebral e Integração \\ Sensório-Motora \\ Instituto de Psiquiatria, UFRJ \\ Rua Sylvio R. Pollis, 300, Casa 2 \\ 22793-395 Rio de Janeiro, RJ \\ Brasil \\ Fax: +55-21-3328-5020 \\ E-mail: andreadeslandes@uol.com.br \\ Research supported by CAPES.}

Received September 29, 2004 Accepted March 2, 2005

\begin{abstract}
Caffeine is the most consumed psychoactive substance in the world. The effects of caffeine have been studied using cognitive and motor measures, quantitative electroencephalography (qEEG) and event-related potentials. However, these methods are not usually employed in combination, a fact that impairs the interpretation of the results. The objective of the present study was to analyze changes in electrophysiological, cognitive and motor variables with the ingestion of caffeine, and to relate central to peripheral responses. For this purpose we recorded event-related potentials and eyes-closed, resting EEG, applied the Stroop test, and measured reaction time. Fifteen volunteers took caffeine $(400 \mathrm{mg})$ or placebo in a randomized, crossover, double-blind design. A significant reduction of alpha absolute power over the entire scalp and of P300 latency at the Fz electrode were observed after caffeine ingestion. These results are consistent with a stimulatory effect of caffeine, although there was no change in the attention (Stroop) test or in reaction time. The qEEG seems to be the most sensitive index of the changes produced by caffeine in the central nervous system since it proved to be capable of detecting changes that were not evident in the tests of cognitive or motor performance.
\end{abstract}

Key words

- Quantitative

electroencephalography

- P300

- Caffeine

- Cognitive and motor

measures

- Psychostimulants

\section{Introduction}

Caffeine is believed to be the most consumed psychostimulant. Different laboratories have investigated its mechanisms of action at the cellular and behavioral level, including inhibition of phosphodiesterase, mobilization of calcium from the sarcoplasmic reticulum, activation of adenylate cyclase and, most notably, as an antagonist of 
adenosine $(1,2)$. Caffeine also exerts modulatory effects on the central nervous system (CNS), especially cognitive functions (1-4). The modulatory responses generated by caffeine were first reported in 1912 based on performance data $(5,6)$, and electrophysiological indices have been used since 1943 (7). However, the effects of this substance on the central and peripheral nervous systems, and the underlying mechanisms, are still under study $(8,9)$. Numerous experiments have shown that caffeine improves cognitive performance by increasing alertness and attention and by reducing fatigue (9-12). The excitatory effects of caffeine are detected in cortical cellular substrates (1$3,9)$. In the CNS, caffeine acts primarily as an adenosine antagonist $(9,13,14)$. However, multiple components are needed to account for the totality of its effects $(1,3)$. Specifically, caffeine enhances the release of neurotransmitters such as catecholamines, serotonin and acetylcholine, associated with vasoconstriction in the brain and vasodilatation in peripheral organs $(5,13)$. Accordingly, it decreases cerebral blood flow (15) and increases brain metabolism. The decrease in cerebral perfusion is probably a consequence of the vasoconstriction caused by catecholamines and inhibition of the vasodilatory effect of adenosine. In this context, quantitative electroencephalography (qEEG) and event-related potentials (ERPs) have been used to monitor the effects of caffeine on brain dynamics (15-19). EEG and ERP data provide a neuropsychophysiological estimation of brain processes which are linked to behavior (20-23). The spectral attributes of the EEG are susceptible to changes under cognitive demands such as attention $(24,25)$. For example, the theta band has been associated with a variety of mental tasks, mainly those that involve continued concentration (26). It has also been demonstrated that the EEG power distribution throughout the cortex is reduced by caffeine ingestion, especially when subjects have their eyes open
$(6,15,27)$. Specifically, alpha absolute power decreases in several brain sites. The effects of caffeine and other methylxanthines on ERPs are controversial $(3,9)$. The ability of the CNS to discriminate and select the appropriate information has been explored by evoked potential techniques $(23,28)$.

Few ERP experiments have attempted to correlate the modulatory effects of caffeine on cognitive processes, attention and arousal in terms of P300 amplitude and latency (16,29-32). A combination of peripheral behavioral responses such as reaction time and attention together with central electrophysiological responses may contribute to the understanding of caffeine's mechanisms of action. Thus, the present investigation combines an attention task, reaction time, qEEG, and ERP to examine the effects of $400 \mathrm{mg}$ caffeine on CNS responses.

\section{Subjects and Methods}

\section{Subjects}

The sample consisted of 15 individuals, 6 males and 9 females ranging in age from 21 to 38 years ( $26 \pm 5$ years), weighing 49 to 83 $\mathrm{kg}(67 \pm 14 \mathrm{~kg})$, moderate caffeine users (i.e., 1-2 cups of coffee per day). All subjects were healthy, right-handed, non-smokers, and free of cognitive deficits, and none of them was using oral contraceptives or any psychoactive or psychotropic substance at the time of the test. To ensure that subjects did not present any impairment in physical or mental health, a questionnaire was applied. The questionnaire also aimed at identifying possible P300 biological determinants, such as food intake, body temperature, fatigue, and drugs, among others. Subjects were not allowed to consume alcoholic or caffeinecontaining beverages during the $10 \mathrm{~h}$ prior to the test, or during the test. They were submitted to a standardized test (Stroop test) for the ability to identify colors (see below) in order to exclude possible defects in color 
vision. Subjects signed a consent form which thoroughly described the experimental procedure. The experiment was approved by the Ethics Committee of the Psychiatric Institute.

\section{Study design and procedures}

Subjects received 400-mg gelatin capsules (caffeine or placebo) on two different occasions in a randomized, double-blind, crossover study. Capsules were manufactured by the University Pharmacy (UFRJ). The procedures consisted of a two-day treatment: placebo and caffeine. The procedures were standardized according to the following routine: electroencephalographic recording, administration of the word color Stroop test and visual ERP. EEG was recorded 30 min after caffeine or placebo ingestion.

\section{Electroencephalogram recording}

The study design respected the norms of the International Pharmaco-EEG group (33). Subjects were seated comfortably in a soundand light-attenuated room, while the EEG was collected from 20 monopolar leads for 5 min (eyes-closed, alert/resting). The data were collected from subjects with their eyes closed in order to record cortical activity in the absence of external stimuli, thereby minimizing visual artifacts. Electrodes were positioned according to the International 10/20 System referred to linked earlobes with ground at FPz. All electrode impedances were kept below $5 \mathrm{k} \Omega$. The signal was amplified with a gain of 22,000 , analogically filtered between $0.01 \mathrm{~Hz}$ (high-pass) and $100 \mathrm{~Hz}$ (lowpass), and sampled at $240 \mathrm{~Hz}$ using a Braintech-3000 ${ }^{\circledR}$ (EMSA-Medical Instruments, Rio de Janeiro, RJ, Brazil) EEG acquisition system. The EEG was recorded by means of the ERP Acquisition software (Delphi 5.0 ${ }^{\circledR}$, Borland-Inprise) developed at the Brain Mapping and Sensorimotor Integration Laboratory, employing the following digital filters: notch $(60 \mathrm{~Hz})$, high-pass of $0.3 \mathrm{~Hz}$ and low-pass of $25 \mathrm{~Hz}$. Visual inspection was used to detect and eliminate artifacts. Eye-movement artifacts were monitored with a bipolar electrode montage using two 9-mm diameter electrodes attached above and on the external canthus of the right eye.

\section{Data analysis}

At least 2 min of artifact-free data were extracted from the EEG record for quantitative analysis. A Matlab 5.3 ${ }^{\circledR}$ (Mathworks Inc., Naticj, MA, USA) routine was implemented to perform a spectral analysis. For each of the 20 monopolar leads, absolute power $\left(\mu \mathrm{V}^{2}\right)$ was computed for the delta $(1.0-3.5 \mathrm{~Hz})$, theta (4.0-7.5 Hz), alpha $(8.0-12.0 \mathrm{~Hz})$, and beta $(13-25 \mathrm{~Hz})$ frequency bands.

\section{Stroop test}

The Stroop test evaluates focused attention and assesses the integrative power of cognitive mechanisms in reaching decisions based upon information from two different modalities (lexical and perceptual) (26). First, subjects were asked to read the names of different colors. Then, to name the printed color of a word denoting a different color; for example, read the word "blue" printed in green (color-word interference). Subjects were asked to perform the task as quickly and as accurately as possible. The Stroop color-naming task is a classic paradigm that elegantly illustrates important concepts such as automaticity and interference. The Stroop paradigm has been in use for over fifty years and has been described as the gold standard of attentional measures (34). The criteria analyzed were execution time and raw score (i.e., number of correct answers).

\section{Event-related potential stimulation protocol}

The ERP recordings were obtained according to standard guidelines (35). During 
the visual task, lights were turned off so that subjects would concentrate exclusively on the monitor screen. A 15 " monitor was placed in front of the volunteer. The visual stimulus was presented on the monitor by the ERP Acquisition software, developed in Delphi 5.0. ERPs were recorded from the 20 electrodes. The task employed the oddball paradigm, in which two stimuli are presented randomly, with one occurring relatively infrequently. Subjects were asked to discriminate target (infrequent) from nontarget or standard stimuli (frequent). In the present experiment, target stimuli were represented by a square and non-target stimuli by a circle, both yellow with black background. In each block, the number of nontarget stimuli preceding each target stimulus was random, with $95 \%$ probability of occurring between 1 and 4 (uniform distribution) and $5 \%$ probability of occurring between 5 and 7. Such distribution resulted in an expected mean value of 2.7 non-target stimuli for each target stimulus (target/non-target ratio of $1 / 2.7$ ), but the real value was randomly different for each test. Thus, for 100 target stimulations, an average of 270 nontarget stimuli were presented. The target/ non-target ratio was $1 / 2.7$. Subjects were instructed to respond as quickly as possible to the target stimulus by pressing a button in a joystick (Model Quick Shot-Crystal CS4281, Quickshot $^{\circledR}$, USA). Epochs regarding target stimuli were considered and averaged only when subjects responded within 150 to $1000 \mathrm{~ms}$. The P300 was identified as the largest positive-going peak amplitude of the waveform within a time window of 250$500 \mathrm{~ms}$ in relation to a pre-stimulus baseline. The baseline was defined as the mean voltage over $120 \mathrm{~ms}$ before the onset of the stimulus. Each individual was subjected to one block of approximately 370 trials. In this protocol, the square was presented 100 times in each block. The stimulus appeared on the screen for $0.75 \mathrm{~s}$, with the same time interval between stimuli.

\section{Reaction time}

The reaction time resulting from the pressing of a button in the joystick after each target stimulus was used as an index of motor performance. Reaction times were subsequently averaged to yield a final value for each subject. Missed stimuli were not considered. Although reaction time is independent of ERP measures, it was used to verify subject alertness during the task.

\section{Statistical analysis}

Preliminary descriptive analyses revealed that none of the evaluated qEEG indices had a normal distribution. To obtain a better approximation to a normal distribution, the absolute power was subjected to a log transformation (log absolute power, LAP). Moreover, to reduce the number of statistical comparisons, LAP values of 12 analyzed electrode sites were grouped into 4 scalp quadrants: right-anterior (mean of $\mathrm{Fp} 2, \mathrm{~F} 4$ and F8), left-anterior (mean of Fp1, F3 and F7), right-posterior (mean of $\mathrm{T} 6, \mathrm{P} 4$ and $\mathrm{O} 2$ ), and left-posterior (mean of T5, P3 and O1). A three-way ANOVA, situation (placebo $v s$ caffeine) by area (right-anterior $v s$ left-anterior $v s$ right-posterior $v s$ left-posterior) by band (delta $v s$ theta $v s$ alpha $v s$ beta) was implemented for LAP data followed by the Scheffé post hoc test. Then, three-way ANOVA followed by the Scheffé post hoc test was applied to analyze possible differences between anterior (Fp1, Fp2, F3, F4, F7, and F8) and posterior (T5, T6, P3, P4, $\mathrm{O} 1$, and $\mathrm{O} 2$ ) regions according to situation and to all frequency bands. Since the alpha band is prominent during eyes-closed EEG recording, a two-way ANOVA was applied to compare anterior and posterior regions in the two experimental conditions (i.e., caffeine and placebo) in this specific frequency band $(8-12 \mathrm{~Hz})$. A paired $t$-test was performed to compare P300 latency and reaction time in the caffeine and placebo condi- 
tions. Wilcoxon's test was used to compare amplitude and Stroop raw score (SRS). The level of significance was set at $\mathrm{P}<0.05$ for all statistical analyses.

\section{Results}

\section{Motor and cognitive results}

Reaction time and Stroop test. Results were compared between the placebo and caffeine conditions. The statistical analyses indicated a nonsignificant shortening in reaction time. The same result was observed for Stroop execution time (SET). The SRS was unchanged in both conditions. Motor and cognitive results are shown in Table 1.

\section{Electrophysiological results}

Visual event-related potential - P300. Caffeine significantly shortened P300 latency at $\mathrm{Fz}$ (caffeine $=313 \pm 61 \mathrm{~ms}$; placebo $=339 \pm 78 \mathrm{~ms}$ ). Moreover, nonsignificant shortenings at $\mathrm{Cz}$ (caffeine $=318 \pm 63 \mathrm{~ms}$; placebo $=340 \pm 77 \mathrm{~ms}$ ) and $\mathrm{Pz}$ (caffeine $=$ $331 \pm 67 \mathrm{~ms}$; placebo $=356 \pm 76 \mathrm{~ms}$ ) were observed. Especially at Fz, P300 latency was shorter with caffeine than with placebo. P300 amplitude was higher in some individuals and lower in others, but the difference between conditions was not significant (caffeine: $\mathrm{Fz}=1.62 \pm 1.84 \mu \mathrm{V}, \mathrm{Cz}=1.63 \pm 1.44$ $\mu \mathrm{V}, \mathrm{Pz}=1.49 \pm 1.46 \mu \mathrm{V}$; placebo: $\mathrm{Fz}=1.72$ $\pm 1.68 \mu \mathrm{V}, \mathrm{Cz}=1.80 \pm 1.30 \mu \mathrm{V}, \mathrm{Pz}=1.83$ $\pm 1.61 \mu \mathrm{V}$ ) (Figure 1).

Quantitative EEG. The variable analyzed for all frequency bands was monopolar absolute power. Figure 2 illustrates LAP values for the four established cortical areas by moment and band. A main effect was found for condition $(F(1,478)=16.59, \mathrm{P}<0.0001)$ and band $(F(3,476)=67.76, \mathrm{P}<0.0001)$, and an interaction between area and band $(F$ $(9,844)=5.45, \mathrm{P}<0.0001)$ was observed Scheffé's post hoc test indicated a difference
Table 1. Cognitive and motor performance variables for the caffeine and placebo conditions

\begin{tabular}{lll}
\hline & Caffeine & Placebo \\
\hline Reaction time (ms) & $382 \pm 53$ & $394 \pm 45$ \\
Stroop execution time (s) & $101 \pm 15$ & $105 \pm 11$ \\
Stroop raw score & $111 \pm 1$ & $111 \pm 1$ \\
\hline
\end{tabular}

Data are reported as means \pm SD.

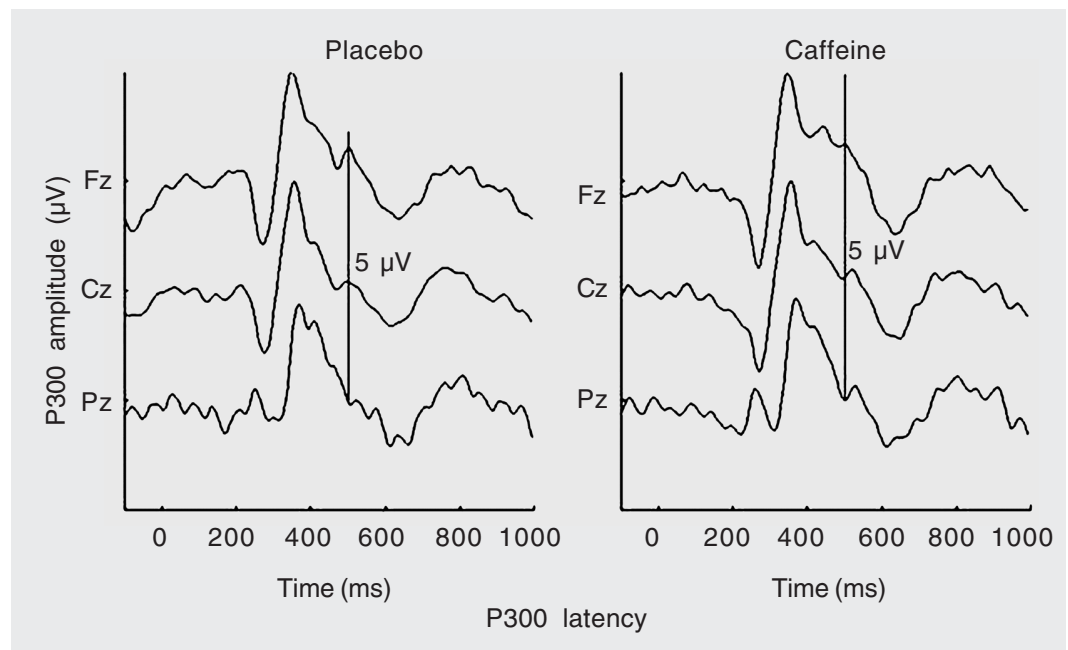

Figure 1. P300 grand average. Event-related potential grand average for latency (ms) and amplitude $(\mu \mathrm{V})$ values for $\mathrm{Fz}, \mathrm{Cz}$ and $\mathrm{Pz}$ electrode sites in the placebo and caffeine conditions.

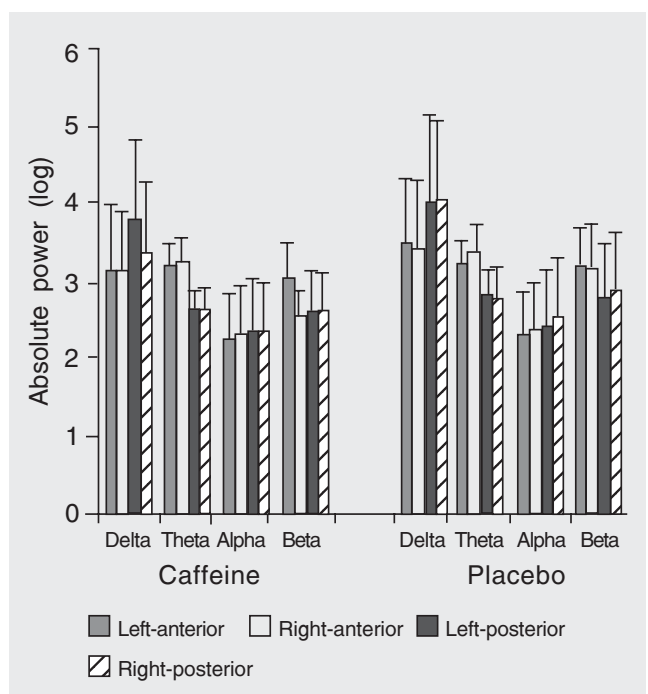

Figure 2. Log absolute power (LAP) values in the caffeine and placebo conditions in the delta, theta, alpha, and beta frequency bands. Data are reported as means \pm SD. Electrodes were applied to four quadrants: rightanterior (Fp1, F3 and F7), leftanterior (Fp1, F3 and F7), rightposterior (T6, P4 and O2) and left-posterior (T5, P3 and O1). A significant LAP reduction was observed in the caffeine condition $(\mathrm{P}<0.05$, three-way ANOVA). 
between alpha and beta in relation to the other bands. A three-way ANOVA performed for two areas (i.e., anterior and posterior), by condition and band, revealed main effects of

Figure 3. Alpha log absolute power values in anterior (frontal, $F p 1, F p 2, F 3, F 4, F 7$, and F8) and posterior (parieto-occipital, T5, T6, P3, P4, O1, and O2) areas, in the two experimental conditions. Data are reported as means \pm SD.
Figure 4. Alpha topography maps. Cortical maps representing mean alpha log absolute power values in the placebo $(A)$ and caffeine (B) conditions $(\mathrm{N}=$ 15). The figure illustrates the $T$ value of the difference between conditions (C). The scales show log (vertical) and T (horizontal) values, which varied between 3 (blue) and 5 (yellow), and 1 (blue) and 3 (orange), respectively.

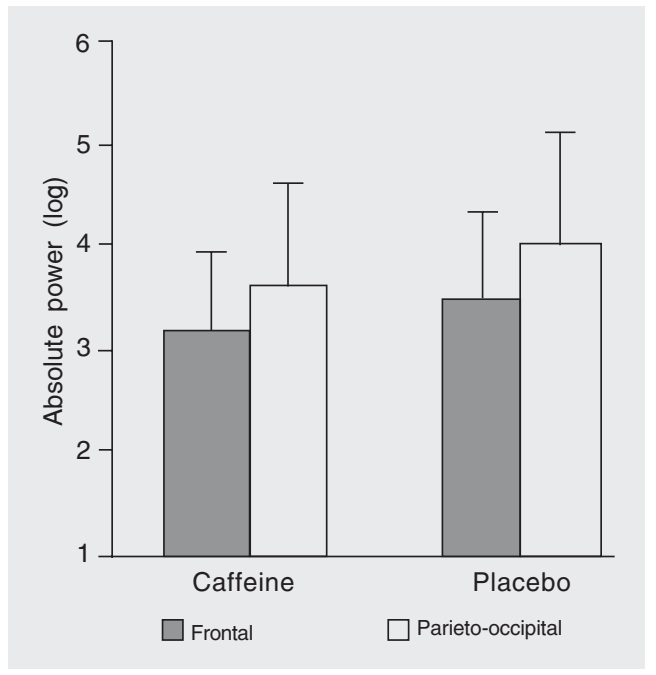

condition $(F(1,478)=16.8, \mathrm{P}<0.0001)$ and band $(F(3,64)=68.73, \mathrm{P}<0.0001)$. A significant LAP reduction was observed in the caffeine condition (total means: caffeine $=2.834 \pm 0.74$ and placebo $=3.076 \pm$ $0.84)$. The analysis also revealed an interaction between area and band $(F(3,952)=$ $15.12, \mathrm{P}<0.0001)$. Scheffe's post hoc test indicated a difference between alpha and beta in relation to the other bands $(\mathrm{P}<0.001)$.

Figure 3 illustrates the results of two-way ANOVA which revealed main effects of condition $(F(1,118)=5.36, \mathrm{P}<0.05)$ and area $(F(1,118)=8.62, \mathrm{P}<0.05)$. No interaction was observed $(\mathrm{P}=0.688)$.

Figure 4 illustrates the mean LAP values for the study sample as a whole in the alpha band $(8-12 \mathrm{~Hz})$. The cortical map shows a significant LAP reduction after caffeine ingestion.

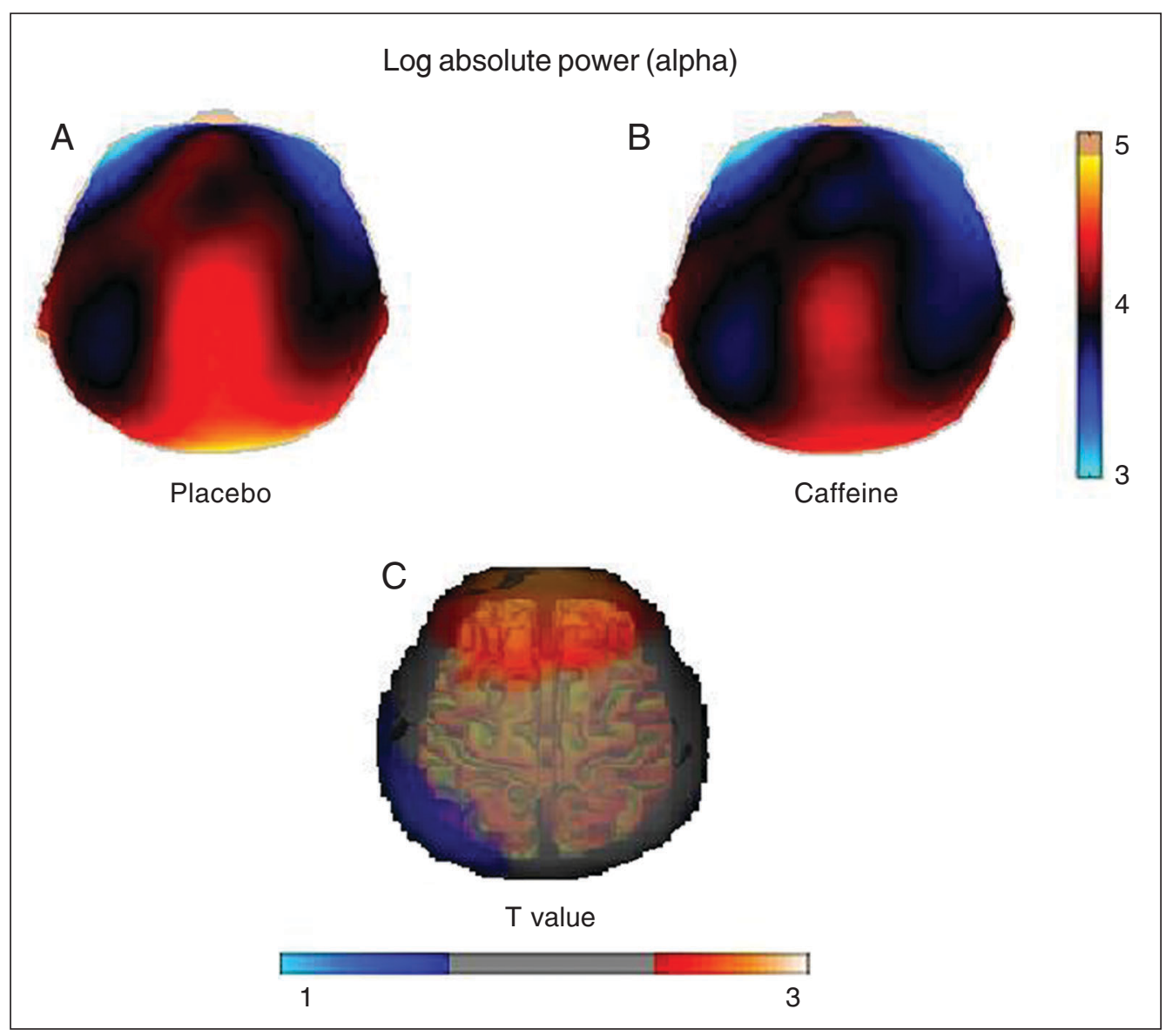




\section{Discussion}

The objective of the present study was to investigate the stimulant effects of caffeine through cognitive, motor and electrophysiological responses. Electrophysiological measures, i.e., visual ERPs and qEEG, revealed significant effects, whereas cognitive and motor measures (Stroop and reaction time) did not. Rarely has it been concluded that electrophysiological measures are better than the cognitive and motor variables to identify the effects of caffeine on brain activity $(9,36)$. Along this line of thought, the present discussion is divided into three sections: cognitive and motor measures, ERPs and $\mathrm{qEEG}$ measures.

\section{Cognitive and motor measures}

The effects of caffeine on human performance have been the focus of several investigations. To explore the action of caffeine on cognitive variables, we exposed the participants to attentional demands. When SET and SRS values were compared between the caffeine and placebo conditions, the results did not indicate significant changes in either parameter, in agreement with data reported by others $(27,34)$. The ability of humans to capture information from the environment is very complex. Sensory detection is the primary step involved in decision-making processes. Sensory data are compared to stored long-term memory, generating perceptual events and, consequently, motor actions. Although the present findings suggest that SET and SRS are not capable of detecting the effects of caffeine on the CNS, there is evidence that caffeine modulates the attentional system, depending on dosage and on subject arousal. The relationship between arousal and cognitive responses takes the form of an inverted U-shaped dose-response curve. Performance is thought to be optimal at intermediate levels of dosage and arousal (3). Although there is no strong agreement on the effects of caffeine on specific cognitive operations, there are indications that caffeine affects the attentional system. In a study performed on rodents, it was shown that caffeine increases concentrations and rates of cerebral norepinephrine, dopamine and serotonin utilization. These results were obtained for neuroanatomic structures of the limbic system and frontal cortex (37). However, in the present investigation no significant differences were found. The same pattern was observed for reaction time. In our investigation, subjects were studied at rest, while the effects of caffeine on behavioral aspects seem to be more evident in a fatigue situation $(27,29)$. Behavioral responses are related to the biological state (i.e., alertness, fatigue, motivation) of the individual. Performance decrement may be due to boredom and fatigue and, thus, be counteracted by caffeine. These effects may be explained by the action of caffeine as an adenosine antagonist. Caffeine seems to delay fatigue by blocking CNS adenosine receptors (38). Adenosine inhibits the release of most brain excitatory neurotransmitters, especially dopamine. Behaviorally, these changes are associated with reduced arousal and increased sleep. In this sense, the stimulant effects of caffeine are diminished when subjects are rested.

\section{Visual event-related potentials}

P300 analyses have indicated a shortening of latency with caffeine ingestion (1719). In the present study, especially at the frontal-cortex level, caffeine improved brain dynamics by reducing latency at Fz. Shorter latencies indicate an increase of informationprocessing speed $(23,39)$. P300 latency is negatively correlated with mental function in normal subjects, with shorter latencies being associated with superior cognitive performance (23). The other areas $(\mathrm{Cz}$ and $\mathrm{Pz})$ showed the same pattern of results, but with no statistical significance. Differences in 
amplitude values were not statistically significant. Some amplitudes decreased and others increased in the caffeine condition. These results could be explained by the wide variability among subjects. Other studies that have analyzed the interaction between ERPs and caffeine are not conclusive. Some studies indicate a shortening of latency $(17,18,29$ 31 , while others show an increase in amplitude $(28,32)$. However, the use of different experimental conditions makes it impossible to compare the results. It is known that P300 is an important measure for the evaluation of cognitive processes, specifically the processes of attention allocation and immediate memory $(23,28,39)$. The increase of P300 amplitude and/or reduction of latency are associated with an enhancement of functional dynamics that takes place after the presentation of the target stimulus. The positive result observed in our experiment, i.e., shorter latencies, suggests that P300 latency is also a sensitive measure for the observation of the effects of caffeine in the brain during a cognitive process. ERP studies indicate that caffeine has an effect on attention, which is independent of specific stimulus characteristics. The main concept is that caffeine modulates information processing through an effect on the perceptual system and output-related processes (9).

\section{Quantitative electroencephalographic analyses}

Among the $\mathrm{qEEG}$ results, the reduction of absolute power, especially in the alpha band, agreed with reports from different laboratories $(9,15,27)$. In this frequency band, CNS depressors are associated with an increase of EEG power and stimulants are associated with a reduction of power over the entire scalp (40). In the present study, a reduction of absolute power was observed on the entire scalp. However, in studies with results similar to ours, the significant results were obtained in subjects tested with their eyes open (15); when subjects closed their eyes, the reduction was not statistically significant. A possible explanation for our results in the eyes-closed condition might be the dosage $(400 \mathrm{mg})$, which was higher than that used in previous studies (200 mg). Similarly, a reduction of power density in the lowest delta band was observed in the sleep EEG. However, there was no reduction of other physiological measures such as heart rate (19).

In this context, we may conclude that the ingestion of caffeine generates a reduction of P300 latency after a visual stimulus and reduces cortical power when individuals are at rest. The literature presents hypotheses that can account for these responses. An increase of neurotransmitter release may be associated with the reduction of $\mathrm{P} 300$ latency, making the transmission of electrical impulses faster. The reduction of EEG power may be related to an increase in brain metabolism, a reduction in cerebral blood flow and vasoconstriction caused by caffeine ingestion.

Finally, we may conclude that the changes produced by caffeine ingestion, although depending on the state of the individual and on dosage, can be more easily quantified through qEEG measures. In a recent review, the authors compared caffeine studies with behavioral measures, EEG data and ERP measures and concluded that the EEG supports the general belief that caffeine acts as a stimulant (9). The present results corroborate this conclusion, since the individuals did not exhibit significant differences in cognitive and motor variables or in P300 amplitude, while there was a significant reduction in EEG absolute power. Possibly, the effects of caffeine ingestion are more evident in the CNS than in the peripheral nervous system. Behavioral effects may be more closely related to peripheral motor processes, with an improvement in performance being more evident in a fatigue situation. Additional studies are needed in order to elucidate the modulatory mechanisms of caffeine in the central and peripheral nervous systems. 


\section{Acknowledgments}

We would like to thank Dr. Martha

\author{
Sorenson, Departamento de Bioquímica, \\ UFRJ, Rio de Janeiro, RJ, Brazil, for revising \\ the manuscript.
}

\section{References}

1. Nehlig A, Daval JL \& Debry G (1992). Caffeine and the central nervous system: Mechanisms of action, biochemical, metabolic, and psychostimulant effects. Brain Research Reviews, 17: 139170.

2. Sawynok J \& Yaksh L (1993). Caffeine as an analgesic adjuvant: a review of pharmacology and mechanisms of action. Pharmacological Reviews, 45: 43-85.

3. James EJ (1991). Caffeine and Health. Academic Press, San Diego, CA, USA.

4. Lieberman $H$ (2001). The effects of ginseng, ephedrine and caffeine on cognitive performance, mood and energy. Nutrition Reviews, 59: 91-102.

5. Hollingworth $\mathrm{HL}$ (1912). The influence of caffeine on the speed and quality of performance in typewriting. Psychological Review, 19: 63-73.

6. Hollingworth $\mathrm{HL}$ (1912). The influence of caffeine on mental and motor efficiency. Archives of Psychology, 22: 1-166.

7. Gibbs FA \& Maltby GL (1943). Effect of electrical activity of the cortex of certain depressant and stimulant drugs: barbiturates, morphine, caffeine, benzedrine and adrenalin. Journal of Pharmacology and Experimental Therapeutics, 78: 1-10.

8. Gevins A, Smith ME \& McEvoy LK (2002). Tracking the cognitive pharmacodynamics of psychoactive substances with combinations of behavioral and neurophysiological measures. Neuropsychopharmacology, 26: 27-39.

9. Lorist MM \& Tops M (2003). Caffeine, fatigue and cognition. Brain and Cognition, 53: 82-94.

10. Johnson-Kohnson M, Kritz-Silverstein D, Barrett-Connor E \& Morton $D$ (2002). Coffee consumption and cognitive function among older adults. American Journal of Epidemiology, 156: 842-850.

11. Tarnopolsky MA (1994). Caffeine and endurance performance. Sports Medicine, 18: 109-125.

12. Nehlig A \& Debry G (1994). Caffeine and sports activity: a review. International Journal of Sports Medicine, 15: 215-223.

13. Laurienti PJ, Field AS, Burdette JH, Maldjian JA, Yen Y \& Moody DM (2002). Dietary caffeine consumption modulates fMRI measures. Neuroimage, 17: 751-757.

14. Notarius C, Atchison D, Rongen G \& Floras J (2001). Effect of adenosine receptor blockade with caffeine on sympathetic response to handgrip exercise in heart failure. American Journal of Physiology, 281: H1312-H1318.

15. Siepmann M \& Kirch W (2002). Effects of caffeine on topographic quantitative EEG. Neuropsychobiology, 45: 161-166.

16. Lorist M, Snel J, Mulder G \& Kok A (1995). Aging, caffeine, and information processing: an event-related potential analysis. Electroencephalography and Clinical Neurophysiology, 96: 453-467.

17. Debrah K, Sherwin R, Murphy J \& Kerr D (1996). Effects of caffeine on recognition of and physiological responses to hypoglycaemia in insulin-dependent diabetes. Lancet, 347: 19-24.

18. Kerr D, Sherwin RS, Pavalkis F, Fayad PB, Sikorski L, Rife F, Tamborlane WV \& During MJ (1993). Effect of caffeine on the recognition of and responses to hypoglycemia in humans. Annals of Internal Medicine, 119: 799-804.

19. Landolt HP, Dijk D, Gaus SE \& Borbély AA (1995). Caffeine reduces low-frequency delta activity in the human sleep EEG. Neuropsychopharmacology, 12: 229-238.

20. Deslandes A, Veiga $H$, Fiszman A, Cagy M, Piedade R \& Ribeiro $P$ (2004). Quantitative electroencephalography (qEEG) to discriminate primary degenerative dementia from major affective disorder (depression). Arquivos de Neuropsiquiatria, 62: 44-50.

21. Veiga $H$, Deslandes $A$, Cagy $M$, Fiszman A, Piedade RA \& Ribeiro $P$ (2003). Neurocortical electrical activity tomography in chronic schizophrenics. Arquivos de Neuropsiquiatria, 61: 712-717.

22. Hillyard SA \& Anllo-Vento L (1998). Event-related brain potentials in the study of visual selective attention. Proceedings of the National Academy of Sciences, USA, 95: 781-787.

23. Polich J \& Herbst K (2000). P300 as a clinical assay: rationale, evolution, and findings. International Journal of Psychology, 38: 319.

24. Egner T \& Gruzelier JH (2004). EEG biofeedback of low beta band components: frequency-specific effects on variables of attention and event-related brain potentials. Clinical Neurophysiology, 115: 131-139.

25. Fernandez T, Herrera W, Harmony $T$ et al. (2003). EEG and behavioral changes following neurofeedback treatment in learning disabled children. Clinical Electroencephalography, 34: 145-152.

26. Smith ME, McEvoy L \& Gevins A (1999). Neurophysiological indices of strategy development and skill acquisition. Cognition Brain Research, 7: 389-404

27. Patat A, Rosenzweig P, Enslen M, Trocherie S, Miget N, Bozon MC, Allain H \& Gandon JM (2000). Effects of a new slow release formulation of caffeine on EEG, psychomotor and cognitive functions in sleep-deprived subjects. Human Psychopharmacology, 15: $153-170$.

28. Polich J \& Kok A (1995). Cognitive and biological determinants of P300: an integrative review. Biological Psychology, 41: 103-146.

29. Seild R, Peryl A, Nicham R \& Hauser E (2000). A taurine and caffeine-containing drink stimulates cognitive performance and well-being. Amino Acids, 19: 635-642.

30. Reeves R, Struve F \& Patrick G (1999). The effects of caffeine withdrawal on cognitive P300 auditory and visual evoked potentials. Clinical Electroencephalography, 30: 24-27.

31. Deslandes A, Veiga H, Cagy M, Piedade R, Pompeu F \& Ribeiro P (2004). Effects of caffeine on visual evoked potential (P300) and neuromotor performance. Arquivos de Neuropsiquiatria, 62: 385390.

32. Kawamura N, Maeda H, Nakamura J, Morita K \& Nakazaa Y (1996). Effects of caffeine on event-related potentials: comparison of oddball with single-tone paradigms. Psychiatry and Clinical Neurosciences, 50: 217-221.

33. Dumermuth $G$, Ferber $G$, Herrmann WM, Hinrichs $H$ \& Künkel $H$ (1987). International Pharmaco-EEG Group (IPEG). Committee on standardization of data acquisition and analysis in pharmaco-EEG investigations. Neuropsychobiology, 17: 213-218. 
34. Edward S, Brice C, Craig C \& Penri-Jones R (1996). Effects of caffeine, practice, and mode of presentation on Stroop task performance. Pharmacology, Biochemistry, and Behavior, 54: 309-315.

35. Picton TW, Bentin S, Berg P et al. (2000). Guidelines for using human event-related potentials to study cognition: recording standards and publication criteria. Psychophysiology, 37: 127-152.

36. Ruijter J, Lorist M, Snel J \& Ruiter M (2000). The influence of caffeine on sustained attention: an ERP study. Pharmacology, Biochemistry, and Behavior, 66: 29-37.

37. Kirch DG, Taylor TR, Gerhardt GA, Benowitz NL, Stephen C \& Wyatt RJ (1990). Effect of chronic caffeine administration on monoamine and monoamine metabolite concentrations in rat brain. Neuropharmacology, 29: 599-602.
38. Davis JM, Zhao Z, Stock HS, Mehl KA, Buggy J \& Hand GA (2003). Central nervous system effects of caffeine and adenosine on fatigue. American Journal of Physiology, 284: R399-R404.

39. Polich J (1999). p300 in clinical applications. In: Niedermeyer E \& Lopes da Silva F (Editors), Electroencephalography: Basic Principles, Clinical Applications and Related Fields. 4th edn. Urban \& Schwarzenberg, Baltimore-Munich, 1073-1091.

40. Kraaier $V$, Van Huffelen AC, Wieneke GH, Van der Worp HB \& Bar PR (1992). Quantitative EEG changes due to cerebral vasoconstriction. Indomethacin versus hyperventilation-induced reduction in cerebral blood flow in normal subjects. Electroencephalography and Clinical Neurophysiology, 82: 208-212. 